La investigación académica sobre fotografía en España. Un análisis de las tesis doctorales entre

$$
2010 \text { y } 2020
$$

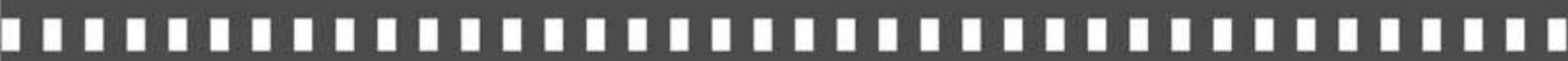

Francisco-José García-Ramos

Isidro Jiménez-Gómez

Artigo recebido em: 07/12/2020

Artigo aprovado em: 21/12/2020 


\title{
La investigación académica sobre fotografía en España. Un análisis de las tesis doctorales entre 2010 y 2020
}

\author{
Academic Research on Photography in Spain. \\ An Analysis of Ph.D Thesis from 2010 to 2020
}

Francisco-José García-Ramos*

Isidro Jiménez-Gómez**

\begin{abstract}
Resumen: Se analiza la producción, temáticas y relaciones entre autoría y dirección de tesis doctorales sobre fotografia en España entre 2010 y 2020 desde una perspectiva de género. Se han aplicado técnicas bibliométricas tomando como fuente principal Dialnet y dos muesttras de tesis en Ciencias Sociales y Humanidades. Las 354 tesis en fotografia (54,52\% mujeres autoras y 62,16\% docentes hombres en roles de dirección), muestran un modelo académico débil para garantizar una constante producción científica.
\end{abstract}

Pabras clave: Tesis doctorales. Fotografía. Universidad. España.

\begin{abstract}
Abstact: This paper analyses the production, themes and relations between authorship and direction of Ph.D. theses on photography in Spain (2010-2020) from a gender perspective. Bibliometric techniques have been applied using Dialnet as the main source and two thesis samples in Social Sciences and Humanities. The 354 theses in photography (54.52\% female authors, and $62.16 \%$ male professors in management roles), show a weak academic model to ensure a constant scientific production.
\end{abstract}

Keywords: Ph.D. theses. Photography. University. Spain.

* Depto. Ciencias de la Comunicación Aplicada, Fac. Ciencias de la Información, Universidad Complutense de Madrid. fjgarciaramos@ucm.es

** Depto. Ciencias de la Comunicación Aplicada, Fac. Ciencias de la Información, Universidad Complutense de Madrid. isidrojimenez@ucm.es 
16 La investigación académica sobre fotografía en España. Un análisis de las tesis doctorales ...

\section{Introducción}

En 1989 se publicaba en España La fotografía y sus posibilidades documentales. Una introducción a su utilización en las Ciencias Sociales. Firmada por Bernardo Riego, MarieLoup Souguez y Miguel Ángel Sánchez, la obra recogía las ideas y reflexiones principales a propósito de la situación de estudios académicos en fotografía en España compartidas en un curso de verano organizado por la Universidad de Cantabria y celebrado en agosto de 1988 en Laredo. En el texto de presentación de la obra, Bernardo Riego — que había puesto en marcha un año antes un Aula de Fotografía supervisada por el Vicerrectorado de Extensión Universitaria - advertía de que el simple hecho de concebir un monográfico otorgando a la fotografía el rango de documento podía suponer un acto académico no exento de cierta controversia.

En los albores de la tercera década del siglo XXI, esta controversia quizás pueda parecer — más aún para las generaciones nativas digitales - sorprendente incluso desde su propio planteamiento. Sin embargo, suponía un verdadero gesto académico cuando apenas había finalizado la década de los 80 del siglo XX y, para entonces, en la universidad española solo se habían defendido nueve tesis doctorales sobre fotografía. Un escaso número al que habría que sumar otras seis más al término de 1989, año en el que se registra el mayor número de tesis doctorales defendidas desde 1976 (SÁNCHEZ-VIGIL; MARCOS-RECIO; OLIVERA-ZALDUA, 2014, p. 4).

En España la incorporación de la fotografía a los estudios 
oficiales se realizó por Real Decreto de 31 de mayo de 1904, tras la reforma del programa de la Escuela Superior de Guerra que incluyó por primera vez la disciplina en la enseñanza pública y privada. En 1916, por Real Orden del Ministerio de Orden Público y Bellas Artes, se creará en Granada la primera Escuela Oficial de Fotografía. A pesar de esto, la fotografía no obtendría el rango de asignatura universitaria hasta 1953 cuando la Escuela Oficial de Periodismo la oferte en el I Curso de Periodistas Gráficos. No obstante, como indica Vega de la Rosa (2007), en España hasta el último tercio del siglo XX no se puede hablar de una auténtica producción académica e historiográfica sobre fotografía "sino más bien de textos que incorporaban referencias tangenciales (tipo memorias), anotaciones de actualidad (tipo artículos y reseñas periodísticas), o análisis puntuales y locales sobre los orígenes de la fotografía en determinada ciudad o región" (VEGA DE LA ROSA, 2007, p. 33). Dado que las dos primeras facultades de Ciencias de la Información se crearon en España en 1971 y las de Bellas Artes comenzaron su andadura en 1978, en los años ochenta la fotografía, tal y como Riego señala en su presentación, todavía no despertaba un interés destacable en los grandes temas a abordar por la investigación académica española:

Otro dato constatable es la poca atención que la fotografía ha tenido como objeto propio de investigación en la Universidad española; siendo hasta el momento muy pocas las tesis y tesinas dedicadas a estudiar sus relaciones con el entorno, primando el uso de imágenes fotográficas como mera "ilustración" de una determinada temática. (RIEGO, 1989, p. 1).

En esta misma línea, en las notas a modo de apéndice de la 
edición española de Historia de la Fotografía de Newhall, publicadas por Gustavo Gili en 1983, Joan Fontcuberta advierte del escaso interés que la fotografía como objeto de estudio había despertado en España, tal y como también se había puesto de manifiesto en las "Jornades Catalanes de Fotografía" (1979-1980). Un desinterés que, como recalca Fontcuberta, había llevado a la paradoja de que algunas de las figuras clave del devenir historiográfico de la fotografía española fueran perfectas desconocidas hasta entonces:

Las razones son múltiples: nula conciencia del valor cultural de la fotografía en la opinión pública, precariedad en la conservación del material fotográfico y escasez de grandes colecciones, negligencia por parte de los organismos competentes de la Administración, endeblez y anquilosamiento de la estructura investigadora de la Universidad española, etc. (FONTCUBERTA, 1983, p. 300).

En cuanto a las causas de la escasa producción científica sobre fotografía vinculada a los programas de doctorado, a las ya apuntadas por Fontcuberta el mismo Riego añadirá los "problemas de acceso a las imágenes derivados de una falta de información sobre su ubicación", problemas metodológicos "que abarcan un amplio abanico, desde la interrelación con el discurso escrito hasta la interpretación, cronología y valoración sociocultural de las imágenes" así como "problemas técnicos derivados de la reproductibilidad de las mismas" (RIEGO, 1989, p. 1-2). Una cuestión que volverá a ser tratada por Riego en la revista Nueva Lente en 1993.

A partir de entonces, el seguimiento de la producción científica universitaria sobre fotografía en España, a pesar de no 
ser muy abundante, ha continuado en trabajos como los de Jones y Baró i Queralt (2000), Vega de la Rosa (2007, 2008), VázquezCasilla (2008), Olivera Zaldua (2014), Vega Pérez (2014), Méndez-Rodríguez y Plaza-Orellana (2017) y, en especial, en los extraordinarios estudios de carácter bibliométrico llevados a cabo por Sánchez-Vigil, Marcos-Recio y Olivera-Zaldua (2014) y Olivera-Zaldua, Sanchez-Vigil y Marcos-Recio (2016). Trabajos que corren paralelos la progresiva incorporación de la fotografía como asignatura obligatoria en nuevos estudios de Grado y Máster ofertados en España a raíz de la implantación del Espacio Europeo de Educación Superior y la entrada en vigor del Real Decreto 1393/2007, de 29 de octubre, por el que se establece la ordenación de las enseñanzas universitarias oficiales. Un contexto que, además, viene acompañado por un creciente interés, tanto por las instituciones nacionales como internacionales, por la recuperación del patrimonio fotográfico (MOREIRO-GONZÁLEZ, 2010) así como por una progresiva presencia de la fotografía en los entornos expositivos y museísticos que ha ido despertando una conciencia de interés hacia la fotografía como manifestación cultural.

Tres décadas después de la publicación de la obra de Riego, Souguez y Sánchez-Gómez y en un contexto marcado por la era digital y la postfotografía, cabe preguntarse nuevamente por la presencia de la fotografía como objeto de estudio en las tesis doctorales de la universidad española, como señala Olivera-Zaldua, Sanchez-Vigil y Marcos-Recio (2016, p. 20), para realizar estudios periódicos sobre las investigaciones en fotografía "al objeto de establecer un observatorio que permitan el seguimiento a la vez que despierten el interés en la materia". En este caso, en lo que se refiere a aquellas tesis 
20 La investigación académica sobre fotografía en España. Un análisis de las tesis doctorales ...

iniciadas superada ya la primera década del siglo XXI y leídas a lo largo de la segunda década del milenio. Tesis doctorales defendidas entre 2010 y 2020 y que conformarían un corpus de investigaciones a través de la cuales poder preguntarse si la llegada de la segunda década del siglo XXI confirma la tendencia ascendente ya observada por Sánchez-Vigil, Marcos-Recio y Olivera-Zaldua (2014).

Unaaproximaciónque, deformamásconcreta, también incluya las relaciones entre temáticas, áreas de conocimiento y universidades aplicando una perspectiva de género en la cuantificación del alumnado que ha desarrollado su investigación doctoral y del profesorado que ha desempeñado funciones de dirección y codirección de tesis. Un enfoque de género que se considera pertinente ahora, más que nunca, que se cumplen 25 años de la Declaración y la Plataforma de Acción de Beijing 95. Y no solo en lo que atañe a estudios específicos sobre mujeres fotógrafas en y através de los medios (GARCÍA-RAMOS, 2017; GARCÍA-RAMOS; JIMÉNEZ-GÓMEZ, 2019). También, y de forma especial, en la aplicación de las recomendaciones de su Sección $\mathrm{J}$ en lo que afecta a la implantación de la perspectiva de género en los estudios universitarios (GARCÍA-RAMOS; ZURIÁNHENÁNDEZ; NÚÑEZ-GÓMEZ, 2020).

\section{Metodología}

Con el fin de analizar la producción y dirección de tesis doctorales sobre fotografía en las universidades españolas entre 2010 y 2020, se optado por una metodología de carácter mixto partiendo de una aproximación bibliométrica al objeto de estudio. La selección 
de las tesis doctorales se ha realizado utilizando como fuente primaria la base de datos Dialnet (https://dialnet.unirioja.es), portal especializado de la Universidad de la Rioja que, como señala León Marin y Magriñá Contreras (2004) es una de fuente bibliográfica consolidada en el ámbito académico español. La razón de optar por Dialnet se debe a la facilidad de su motor de búsqueda y a la posibilidad que ofrece para acceder a través de ella a los contenidos de otras bases de datos sobre las tesis depositadas en las universidades españolas, entre ellas Teseo, del Ministerio de Educación, Cultura y Deporte. Algo que ha facilitado complementar la recogida de datos a través de repositorios propios de universidades españolas como fuentes secundarias: ACCEDA, ADDI, Archivo Digital UPM, Biblos-e Archivo, DIGIBUG, Dipòsit Digital de la UB, DDD, e-Archivo, E-Prints Complutense, e-Repositori UPF, GREDOS, Helvia, idUS, Investigo, MINERVA, RediUMH, Repositori UIB, RIULL, RIUMA, RiuNet, RODERIC, RUC, RUIdeR, TDX, DDD, UCrea y ZAGUÁN.

El motor de búsqueda de Dialnet permite seleccionar por término acotando dicha búsqueda a tesis doctorales en una horquilla temporal macada. La selección de resultados incorpora todas las referencias que incorporen el término de búsqueda ya sea en el título, palabras clave o resumen de la tesis doctoral. De esta manera, el filtrado de tesis ha requerido la lectura del resumen de todas ellas así como la consulta del índice de contenidos de la propia tesis a través del repositorio de la universidad donde está alojada. En la tarea de filtrado, se han descartado aquellas tesis en las que la incorporación del término "fotografía" obedezca a: una simple fuente secundaria sin que constituya el eje central de la investigación, "se consultaron 
22 La investigación académica sobre fotografía en España. Un análisis de las tesis doctorales ...

fuentes documentales de la época, consistentes en los periódicos, gacetas oficiales y fotografías"; un mero instrumento de recogida de información, "se tomaron fotografías y se midió el tamaño" o "para ello se han recurrido a las técnicas de video y fotografía"; una mención como ejemplo de la historia de los medios de comunicación, “esta dinámica de observación-reflejo se repite a través de la historia con la fotografía, el cine, la televisión y, por último, con la informática"; una simple alusión en la enumeración de géneros artísticos "experimentación con diferentes géneros, formatos $\mathrm{y}$ soportes (poesía, narrativa, teatro, ensayo, videocreación, fotografía, performance, artes plásticas y música...)"; la dirección de fotografía en el cine, "la contribución de los colaboradores artísticos más importantes del cineasta, como los directores de fotografía y diseño, compositores, guionistas"; o, entre otras, al empleo del término para referirse a una panorámica o visión general sobre un tema en concreto, "esta tesis pretende fotografiar las múltiples formas en que los miembros de la familia expresan las prácticas sociales".

Tras este proceso de selección, se ha realizado una intensa labor documental para completar, contrastar y normalizar otros datos como los nombres de personas, departamentos, centros e instituciones. Con el fin de conseguir el mayor número de referencias se ha hecho una búsqueda partiendo del término fotografía* y una complementaria con el prefijo foto*, que integra otros descriptores de interés como fototipia, daguerrotipo, fotograbado, fotomecánica y fotoperiodismo.

Finalmente, se optó por el criterio de búsqueda fotografía* dando como resultado un total de 2062 tesis doctoral en términos totales. Acotando la búsqueda a la horquilla comprendida entre 2010 
y 2020, se establece un registro inicial de 1315 tesis doctorales a revisar. Tras la comprobación manual de cada una de ellas, se seleccionaron todas aquellas cuya temática abordase cualquier cuestión relativa al amplio campo de la fotografía como elemento articulador de la investigación independientemente del área de conocimiento, descartándose todas aquellas en donde la fotografía aparece, como ya advertía Riego (1989), como simple elemento instrumental, ilustrativo o sin mayor relevancia o protagonismo como objeto de estudio en sí mismo. Finalizado el proceso de comprobación y selección, se establece un muestra final de 354 tesis doctorales sobre fotografía defendidas en la universidad española entre 2010 y 2020 que conforma el objeto de estudio. Por último, una vez obtenida toda la información, se han establecido las siguientes categorías de análisis de cada tesis: autoría y género, temática, área de conocimiento, centro, universidad, dirección y codirección de la tesis y género.

De cara a realizar un análisis comparado y poder contextualizar mejor las características específicas de las tesis sobre fotografía, se han elaborado otras dos muestras complementarias que recogen de forma aleatoria tesis doctorales defendidas entre 2010 y 2020. La primera la configura una muestra aleatoria de tesis del área de las Humanidades y la segunda de ellas del área de Ciencias Sociales. La muestra de tesis sobre Humanidades consta de 657 tesis doctorales, siendo muestras representativas, con un $5 \%$ de margen de error y el 99\% de nivel de confianza, de las 51.401 tesis doctorales sobre esta temática alojadas en Dialnet en diciembre de 2020 (Tabla 1). La muestra aleatoria elaborada a partir de tesis sobre Ciencias Sociales es representativa de las 63.430 tesis doctorales sobre esta temática 
24 La investigación académica sobre fotografía en España. Un análisis de las tesis doctorales ...

(Tabla 1).

Tabla 1 - Frecuencia de la temática de las tesis doctorales sobre Humanidades y Ciencias Sociales alojadas en Dialnet a diciembre de 2020

\begin{tabular}{|c|c|c|c|}
\hline $\begin{array}{c}\text { Temática } \\
\text { Tesis Doctorales } \\
\text { en Humanidades }\end{array}$ & $\begin{array}{c}\text { Número de Tesis } \\
\text { Doctorales }\end{array}$ & $\begin{array}{c}\text { Temática } \\
\text { Tesis Doctorales } \\
\text { en Ciencias Sociales }\end{array}$ & $\begin{array}{c}\text { Número de Tesis } \\
\text { Doctorales }\end{array}$ \\
\hline Historia & 19618 & Antropología & 3735 \\
\hline Lingüística & 9461 & Demografía & 2107 \\
\hline Artes & 15743 & Ciencias Económicas & 17681 \\
\hline Ética & 1201 & C.C. Jurídicas y Derecho & 10528 \\
\hline Filosofia & 5378 & Pedagogía & 10356 \\
\hline & & Ciencia Política & 7967 \\
\hline Total & & Sociología & 11056 \\
\hline
\end{tabular}

Fuente: Elaboración propia.

En cuanto a las temáticas de las tesis sobre fotografía se ha partido de las definidas por investigaciones previas como las de Sánchez-Vigil, Marcos-Recio y Olivera-Zaldua (2014), que establecen siete categorías generales: Arte, Autores (vida y obra), Comunicación, Documentación, Historia, Sociología, y Técnica y Tecnología. No obstante, a la hora de realizar un análisis cualitativo de las mismas, se ha tenido en cuenta algunas matizaciones basadas en la proliferación de temáticas en fotografía sobre: archivo $\mathrm{y}$ memoria en las categorías de Arte, Documentación y Autores (vida y obra); arquitectura en la categoría Arte; educomunicación en Comunicación; enfoques más próximos a la etnografía y antropología que complementan la categoría Sociología; y propaganda, memoria histórica, historia urbana, del paisaje y del territorio en lo que se refiere a la categoría Historia. 


\section{Resultados}

Las 354 tesis doctorales sobre fotografía suponen una pequeña proporción del total de las tesis alojadas por Dialnet a diciembre de 2020, representando un $0,69 \%$ de las 51.401 tesis doctorales sobre humanidades y un $0,13 \%$ de las 278.409 tesis doctorales catalogadas por el portal. Como muestra la Figura 1, entre 2015 y 2017 se produce un aumento considerable en la defensa de tesis doctorales en el área de Humanidades y Ciencias Sociales.

Fig. 1 - Evolución gráfica de las tesis doctorales sobre fotografía, Humanidades y Ciencias Sociales en el periodo 2010-2020

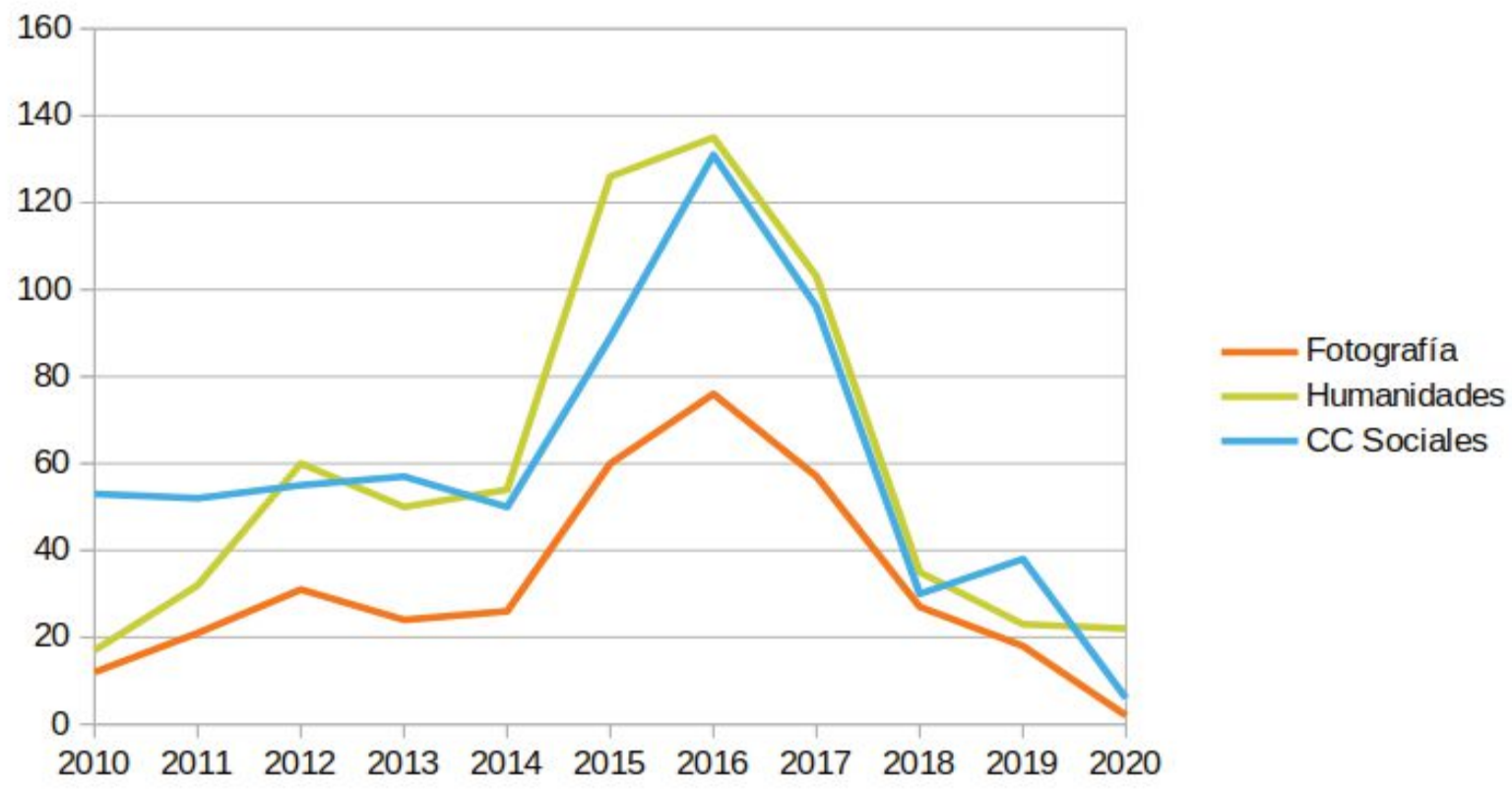

Fuente: Elaboración propia según datos de Dialnet.

Este aumento del número de defensas de tesis coincide en el tiempo con los últimos años del plazo de extinción de los programas de doctorado vigentes en esa fecha y la reforma de los planes de tercer ciclo de la universidad española según el marco establecido por el Plan Bolonia y atendiendo al Real Decreto 99/2011, de 28 de 
26 La investigación académica sobre fotografía en España. Un análisis de las tesis doctorales ...

enero, por el que se regulan las enseñanzas oficiales de doctorado en España. No obstante, tal y como muestra la Tabla 2, el caso de las tesis sobre fotografía y pese a detectarse una marcada tendencia ascendente entre 2015 y 2017, este fenómeno es menos acusado respecto a las cifras la muestra de Humanidades y Ciencias Sociales. Algo que señala cierta estabilidad en la elaboración de tesis doctorales sobre esta temática.

Tabla 2 - Comparativa de tesis doctorales sobre fotografía, Humanidades y Ciencias Sociales en el periodo 2010-2020

\begin{tabular}{|c|c|c|c|c|c|c|}
\hline AÑO & Fotografía & $\mathbf{\%}$ & Humanidades & $\mathbf{\%}$ & CC Sociales & $\mathbf{\%}$ \\
\hline 2020 & 2 & $0,56 \%$ & 22 & $3,35 \%$ & 6 & $0,91 \%$ \\
\hline 2019 & 18 & $5,08 \%$ & 23 & $3,50 \%$ & 38 & $5,78 \%$ \\
\hline 2018 & 27 & $7,63 \%$ & 35 & $5,33 \%$ & 30 & $4,57 \%$ \\
\hline 2017 & 57 & $16,10 \%$ & 103 & $15,68 \%$ & 96 & $14,61 \%$ \\
\hline 2016 & 76 & $21,47 \%$ & 135 & $20,55 \%$ & 131 & $19,94 \%$ \\
\hline 2015 & 60 & $16,95 \%$ & 126 & $19,18 \%$ & 89 & $13,55 \%$ \\
\hline 2014 & 26 & $7,34 \%$ & 54 & $8,22 \%$ & 50 & $7,61 \%$ \\
\hline 2013 & 24 & $6,78 \%$ & 50 & $7,61 \%$ & 57 & $8,68 \%$ \\
\hline 2012 & 31 & $8,76 \%$ & 60 & $9,13 \%$ & 55 & $8,37 \%$ \\
\hline 2011 & 21 & $5,93 \%$ & 32 & $4,87 \%$ & 52 & $7,91 \%$ \\
\hline 2010 & 12 & $3,39 \%$ & 17 & $2,59 \%$ & 53 & $8,07 \%$ \\
\hline Total & 354 & $100,00 \%$ & 657 & $100,00 \%$ & 657 & $100,00 \%$ \\
\hline
\end{tabular}

Fuente: Elaboración propia según datos de Dialnet.

En cuanto a las universidades donde se han defendido más tesis doctorales sobre fotografía, destaca la Universidad Complutense de Madrid, con un 17,23\%. Un porcentaje superior al que esta misma universidad presenta en la muestra aleatoria de tesis del área de Humanidades $(11,26 \%)$ o en las del área de Ciencias Sociales $(11,28 \%)$, tal y como se muestra en la Tabla 3 . Atendiendo a los datos obtenidos, las universidades con mayor número de tesis doctorales de Humanidades son las que también presentan un mayor número 
de tesis sobre fotografía. Las diferencias con las universidades destacadas en las tesis sobre Ciencias Sociales son mayores. En todo caso, entre la muestra de tesis del área de Humanidades no aparecen tres universidades que, por el contrario, sí destacan en el número de tesis doctorales sobre fotografía. Es el caso de la Universitat Pompeu Fabra, la Universidad de La Laguna y la Universidade de Vigo, lo que hace pensar que cuentan con asignaturas y profesorado que favorece la elección de esta temática. Respecto a las facultades y centros universitarios donde son más frecuentes las tesis doctorales sobre fotografía, destacan en primer lugar las facultades de Bellas Artes y Diseño (103), seguidas de las de Ciencias de la Información y Comunicación (68), Geografía, Historia e Historia del Arte (54), E.T.S. Arquitectura (22), Educación (8), facultades de Humanidades y Filología y Letras (7). 
28 La investigación académica sobre fotografía en España. Un análisis de las tesis doctorales ...

Tabla 3 - Universidades donde se defendieron las tesis doctorales entre 2010-2020

\begin{tabular}{|c|c|c|c|c|c|c|c|c|}
\hline $\begin{array}{l}\text { Tesis sobre } \\
\text { fotografía }\end{array}$ & $\mathrm{N}^{\circ}$ & $\%$ & $\begin{array}{c}\text { Tesis en el área } \\
\text { de Humanidades }\end{array}$ & $\mathrm{N}^{\circ}$ & $\%$ & $\begin{array}{l}\text { Tesis en el área } \\
\text { CC. Sociales }\end{array}$ & $\mathrm{N}^{\circ}$ & $\%$ \\
\hline $\begin{array}{l}\text { U. Complutense de } \\
\text { Madrid }\end{array}$ & 61 & $\begin{array}{c}17,23 \\
\%\end{array}$ & $\begin{array}{l}\text { U. Complutense de } \\
\text { Madrid }\end{array}$ & 74 & $\begin{array}{c}11,26 \\
\%\end{array}$ & $\begin{array}{l}\text { U. Complutense de } \\
\text { Madrid }\end{array}$ & 74 & $\begin{array}{c}11,28 \\
\%\end{array}$ \\
\hline U. de Barcelona & 30 & $8,47 \%$ & U. de Granada & 72 & $\begin{array}{c}10,96 \\
\%\end{array}$ & U. de Málaga & 29 & $4,42 \%$ \\
\hline U. de Granada & 26 & $7,34 \%$ & U. de Sevilla & 69 & $\begin{array}{c}10,50 \\
\%\end{array}$ & UNED & 29 & $4,42 \%$ \\
\hline $\begin{array}{l}\text { U. Politècnica de } \\
\text { València }\end{array}$ & 22 & $6,21 \%$ & U. de Barcelona & 43 & $6,54 \%$ & $\begin{array}{l}\text { U. Autònoma de } \\
\text { Barcelona }\end{array}$ & 29 & $4,42 \%$ \\
\hline U. de Sevilla & 22 & $6,21 \%$ & $\begin{array}{l}\text { U. Politècnica de } \\
\text { València }\end{array}$ & 33 & $5,02 \%$ & U. del País Vasco & 27 & $4,12 \%$ \\
\hline U. Politécnica de Madrid & 13 & $3,67 \%$ & U. de Zaragoza & 28 & $4,26 \%$ & U. de Sevilla & 25 & $3,81 \%$ \\
\hline U. Pompeu Fabra & 12 & $3,39 \%$ & U. Autónoma de Madrid & 27 & $4,11 \%$ & U. de Granada & 23 & $3,51 \%$ \\
\hline U. de La Laguna & 11 & $3,11 \%$ & U. de València & 22 & $3,35 \%$ & U. de Alicante & 23 & $3,51 \%$ \\
\hline U. de Vigo & 10 & $2,82 \%$ & U. Politécnica de Madrid & 21 & $3,20 \%$ & U. de València & 22 & $3,35 \%$ \\
\hline U. de Málaga & 9 & $2,54 \%$ & U. del País Vasco & 21 & $3,20 \%$ & U. de Salamanca & 21 & $3,20 \%$ \\
\hline U. de Alicante & 9 & $2,54 \%$ & U. de Oviedo & 18 & $2,74 \%$ & U. de Zaragoza & 21 & $3,20 \%$ \\
\hline U. Autónoma de Madrid & 8 & $2,26 \%$ & U. de Málaga & 17 & $2,59 \%$ & U. de La Laguna & 19 & $2,90 \%$ \\
\hline $\begin{array}{l}\text { U. Autònoma de } \\
\text { Barcelona }\end{array}$ & 7 & $1,98 \%$ & U. de Murcia & 14 & $2,13 \%$ & $\begin{array}{l}\text { U. de Santiago } \\
\text { Compostela }\end{array}$ & 18 & $2,74 \%$ \\
\hline U. de Zaragoza & 7 & $1,98 \%$ & $\begin{array}{l}\text { U. Autònoma de } \\
\text { Barcelona }\end{array}$ & 13 & $1,98 \%$ & U. Autónoma de Madrid & 17 & $2,59 \%$ \\
\hline U. de Salamanca & 7 & $1,98 \%$ & $\begin{array}{l}\text { U. de Santiago } \\
\text { Compostela }\end{array}$ & 13 & $1,98 \%$ & U. Rey Juan Carlos & 17 & $2,59 \%$ \\
\hline
\end{tabular}

Fuente: Elaboración propia según datos de Dialnet.

Sobre el número de profesorado implicado en la tutoriación de tesis sobre fotografía entre 2010 y 2020, ya desempeñen labores de dirección o de codirección, se cuantifican un total de 474 docentes y una media de 1,33 docentes por tesis, prácticamente las mismas cifras en el caso de las tesis doctorales del área de Humanidades (con 1,36 docentes por tesis) y Ciencias Sociales (con 1,39 docentes por tesis). No obstante, poniendo el foco en la codirección de tesis doctorales, los datos varían levemente entre las tesis de fotografía, donde hay un mayor número de trabajos dirigidos por dos o más docentes y las dos muestras analizadas. En el caso de las tesis sobre fotografía la codirección se da en 144 casos, un 40,68\%, 
en comparación con el 35,01\% que se observa en la muestra de Humanidades y el 37,14 \% de Ciencias Sociales. Por otro lado, en cuanto a las tesis sobre fotografía, el $87 \%$ del profesorado tan solo ha dirigido o codirigido una tesis doctoral sobre fotografía. Atendiendo a los datos ofrecidos por Dialnet, la Tabla 4 muestra los 14 docentes con al menos 3 direcciones/codirecciones de tesis sobre fotografía. Tan sólo María Dolors Tapias, Francisco García García y Carlos Ameller han participado en, al menos, 5 tesis doctorales sobre esta temática.

Tabla 4 - Docentes en la dirección y/o codirección de tres o más tesis doctorales sobre fotografía entre 2010-2020

\begin{tabular}{|l|c|c|l|c|c|}
\hline \multicolumn{1}{|c|}{ Docente } & Univ. & $\mathbf{N}^{\circ}$ Tesis & \multicolumn{1}{c|}{ Docente } & Univ. & ఎ $^{\circ}$ Tesis \\
\hline María Dolors Tapias Gil & UB-UPV & 8 & Antonio Á. Ruiz Rodríguez & UGR & 3 \\
\hline Francisco García García & UCM & 7 & Asunción Domeño Martínez & UNAV & 3 \\
\hline Carlos Ameller Ferretians & UB & 5 & Fernando Hernández Hdz. & UB & 3 \\
\hline Joaquin Roldán Ramirez & UGR & 4 & Juan Miguel Sánchez Vigil & UCM & 3 \\
\hline Josep Benlloch Serrano & UPV & 4 & Miquel Planas i Rosselló & UB & 3 \\
\hline Nekane Parejo Giménez & UCO -UMA & 4 & Ricardo Marín Viadell & UGR & 3 \\
\hline$M^{2}$ Santos García Felguera & UPF-UB-UC & 4 & Sagrario Aznar Almazán & UNED-UCM & 3 \\
\hline
\end{tabular}

UB (Universitat de Barcelona); UPV (Universitat Politècnica de València); UCM (Universidad Complutense de Madrid); UGR (Universidad de Granada); UCO (Universidad de Córdoba); UMA (Universidad de Málaga); UNAV (Universidad de Navarra); UPF (Universidad Pompeu Fabra); UC (Universidad de Cantabria); UNED (Universidad Nacional de Educación a Distancia).

Fuente: Elaboración propia según datos de Dialnet.

En lo que se refiere a la temática que abordan las tesis doctorales sobre fotografía, tal y como indica la Tabla 5, las categorías Arte, con 117 tesis, y Autores (vida y obra), con 57 tesis, representan en conjunto el 49,15\% del total de producción académica de la última década. No obstante, tras la categoría Arte, la segunda que presenta un mayor número de tesis doctorales entre 2010 y 2020 será Comunicación, con 88 tesis doctorales, lo que representa un 24,85\% 
30 La investigación académica sobre fotografía en España. Un análisis de las tesis doctorales ...

del total.

En un análisis más detallado de cada una de las categorías, es destacable que en la que se refiere a Arte, 16 tesis $(13,67 \%)$ abordan cuestiones que afectan a la fotografía y la arquitectura y 9 de ellas $(7,69 \%)$ a cuestiones que intersecan la fotografía con el arte, las prácticas de archivo y la memoria. En este sentido, excepcional será la inclusión en esta categoría de una tesis doctoral que aborda fotografía y arteterapia. Respecto a la categoría Autores (vida y obra), tercera en número de tesis doctorales defendidas en este periodo, a pesar de que muchas de ellas abordan de forma indirecta prácticas de archivo, 5 de ellas articularán su desarrollo de forma destacable en este ámbito. Aunque esta cifra solo suponga un 8,77\% del total, la práctica de archivo y la catalogación de obra inédita se observa como transversal a esta categoría.

Tabla 5 - Categorías temáticas de las tesis sobre fotografía defendidas entre 2010-2020.

\begin{tabular}{|c|c|c|}
\hline Categorías temáticas & $\mathbf{N}^{\mathbf{0}}$ Tesis & $\mathbf{\%}$ \\
\hline Arte & 117 & $33,05 \%$ \\
\hline Comunicación & 88 & $24,85 \%$ \\
\hline Autores (vida y obra) & 57 & $16,10 \%$ \\
\hline Historia & 32 & $9,03 \%$ \\
\hline Técnica y Tecnología & 31 & $8,75 \%$ \\
\hline Sociología y Antropología & 17 & $4,80 \%$ \\
\hline Documentación y Archivo & 12 & $3,38 \%$ \\
\hline Total & 354 & \\
\hline
\end{tabular}

Fuente: Elaboración propia en base a Dialnet y repositorios de tesis de universidad.

En Comunicación, segunda categoría en número de tesis, serán destacables sus 24 tesis $(27,27 \%)$ que abordan la fotografía desde el ámbito de la educomunicación. El resto, la conforman aproximaciones más minoritarias como las que abordan su 
marco jurídico y legal ( 2 tesis) y otros aspectos que atraviesan la comunicación con la psicología y la sociología de la imagen (2 tesis). Historia será la cuarta categoría en número con 32 tesis doctorales $(9,03 \%)$. No obstante, el número de tesis con un enfoque puramente historiográfico sobre la propia fotografía, 5 tesis, solo supondrá un $15,62 \%$ del total en esta categoría. La relación entre fotografía e historia desde una aproximación que la sitúa como dispositivo de memoria será representada por 10 tesis $(31,25 \%)$ a las que hay que sumar otras 4 tesis $(12,5 \%)$ que la atraviesan desde la historia y la propaganda política. Significativas serán las 14 tesis, que suponen un significativo 43,75\% del total de la categoría, cuyo enfoque se basa en las relaciones entre fotografía y la historia de las ciudades, del urbanismo, del paisaje y del territorio.

Con 31 tesis doctorales (un 8,75\%), la categoría Técnica y Tecnología presenta un porcentaje similar al de Historia. Por su parte, Sociología y Antropología (17 tesis) y Documentación (12 tesis) con un $4,80 \%$ y un 3,38\% respectivamente serán las categorías con menor número de tesis defendidas en este periodo. En cuanto a Sociología y Antropología, 12 de ellas (70,58\%) presentan un enfoque más sociológico respondiendo las 5 restantes a una aproximación más orientada a la antropología y la etnografía. Por último, con 12 tesis, la categoría Documentación y Archivo supondrá un 3,38\% de la cifra total. Como ya se observa en otras categorías, también en aquí será reseñable que el $25 \%$ de las tesis, 3 de ellas, presentarán un enfoque atravesado por las prácticas de archivo y memoria.

El análisis cuantitativo por género aplicado a la autoría de las tesis doctorales y al profesorado que ha desempeñado tareas de dirección y codirección, muestra un reparto prácticamente equitativo 
32 La investigación académica sobre fotografía en España. Un análisis de las tesis doctorales ...

entre hombres y mujeres en las tesis de fotografía respecto a las dos muestra de referencia del área de Humanidades y de Ciencias Sociales. No obstante, en estos dos últimos casos el dato de hombres supera al de mujeres autoras (Tabla 6). Una relación que, manteniendo una proporcionalidad similar, se invierte en las producción de tesis doctorales sobre fotografía donde se observa un ligero predominio de mujeres autoras: un $54,52 \%$ frente a un $45,48 \%$.

Tabla 6 - Frecuencia de autores de las tesis doctorales de las tres muestras según género

\begin{tabular}{|l|c|c|c|c|c|c|}
\hline Autoría & Fotografia & \% & Humanidades & \% & CC Sociales & \% \\
\hline Hombres: & 161 & $45,48 \%$ & 329 & $50,08 \%$ & 344 & $52,36 \%$ \\
\hline Mujeres: & 193 & $54,52 \%$ & 328 & $49,92 \%$ & 313 & $47,64 \%$ \\
\hline Total: & 354 & $100,00 \%$ & 657 & $100,00 \%$ & 657 & $100,00 \%$ \\
\hline
\end{tabular}

Fuente: Elaboración propia en base a Dialnet 2010-2020.

La mayor presencia de la mujer en la autoría de tesis vinculadas a la fotografía también se constata en el conjunto del profesorado, donde el porcentaje de mujeres en la dirección y codirección es mayor en el caso de las tesis doctorales sobre fotografía que en las tesis de las muestras del área de Humanidades y Ciencias Sociales (Tabla 7). No obstante, aunque porcentualmente las mujeres directoras y codirectoras superen al de las dos muestras de referencia, en las tesis de fotografía que se han defendido entre 2010 y $2020 \mathrm{su}$ número es significativamente menor, 180 mujeres $(37,97 \%)$ frente a 294 hombres (62,03\%). Más todavía al considerar que de los siete docentes con más de cuatro tesis dirigidas o codirigidas, tres de ellas son mujeres y la que encabeza la lista en número es una mujer: María Dolors Tapias Gil (Tabla 4). 
Tabla 7 - Frecuencia del total directores/as y codirectores/as de las tesis doctorales según género y frecuencia de primeros/as directores/as de las tesis doctorales según género

\begin{tabular}{|l|c|c|c|c|c|c|}
\hline $\begin{array}{l}\text { Dirección } \\
\text { y codirección }\end{array}$ & Fotografia & $\%$ & Humanidades & \% & CC. Sociales & $\%$ \\
\hline Hombres & 294 & $62,03 \%$ & 636 & $70,82 \%$ & 635 & $69,32 \%$ \\
\hline Mujeres & 180 & $37,97 \%$ & 262 & $29,18 \%$ & 281 & $30,68 \%$ \\
\hline & 474 & $100,00 \%$ & 898 & $100,00 \%$ & 916 & $100,00 \%$ \\
\hline Dirección & & & & & & \\
\hline Hombres & 229 & $64,69 \%$ & 471 & $71,69 \%$ & 482 & $73,36 \%$ \\
\hline Mujeres & 125 & $35,31 \%$ & 186 & $28,31 \%$ & 175 & $26,64 \%$ \\
\hline
\end{tabular}

Fuente: Elaboración propia en base a Dialnet y repositorios de tesis de universidad 2010-2020.

Esta desigualdad que se constata en los tres casos será todavía más visible al poner el foco en las mujeres que aparecen como directoras o en el primer lugar en la dirección: 125 mujeres $(35,31 \%)$ frente a 229 hombre (64,69\%). Sin embargo, será en las tesis sobre fotografía donde la diferencia sea menos acusada que en la muestras de referencia, donde no alcanzan el 30\% en ninguno de los dos casos: un 28,31\% en el caso de las tesis en el área de Humanidades y un 26,64\% en las tesis de Ciencias Sociales.

En lo que se refiere a la vinculación entre el género de la autoría y el profesorado en la dirección de la tesis doctoral, la relación "autor hombre y director hombre" es más frecuente en las tesis en Ciencias Sociales y de Humanidades que en las tesis sobre fotografía (Tabla 11). Asimismo, es especialmente significativa la relación entre "autoras mujeres y directoras mujeres": en las tesis sobre fotografía alcanza el $21,75 \%$ respecto al $15,22 \%$ que se observa en las tesis de Ciencias Sociales y el 16,59\% de las tesis de la muestra de Humanidades. Por otro lado, en lo que afecta a las tesis sobre fotografía, los doctorandos optarán de forma mayoritaria 
34 La investigación académica sobre fotografía en España. Un análisis de las tesis doctorales ...

por directores hombres, en un 36,44\% de los casos frente al 9,04\% que optan por una mujer directora. La decisión de las doctorandas, sin embargo, no presentará una descompensación tan marcada: en el $32,77 \%$ de las tesis se decantan por un hombre como director y en un $21,75 \%$ por una mujer. En cualquier caso, en las tesis sobre fotografía la relación "autor hombre y directora mujer" será mucho menos frecuente, 32 tesis $(9,04 \%)$, que la que la relación "autora mujer y directora mujer", que asciende a 77 tesis $(21,75 \%)$ en el periodo objeto de estudio.

Tabla 11 - Vinculación según género de autores/as y directores/as de las tesis doctorales

\begin{tabular}{|l|c|c|c|c|c|c|}
\hline Tesis Doctorales & Fotografía & $\%$ & Humanidades & $\%$ & CC Sociales & $\%$ \\
\hline $\begin{array}{l}\text { Autor Hombre } \\
\text { Director Hombre }\end{array}$ & 129 & $36,44 \%$ & 252 & $38,36 \%$ & 269 & $40,94 \%$ \\
\hline $\begin{array}{l}\text { Autor Hombre } \\
\text { Directora Mujer }\end{array}$ & 32 & $9,04 \%$ & 77 & $11,72 \%$ & 75 & $11,42 \%$ \\
\hline $\begin{array}{l}\text { Autora Mujer } \\
\text { Directora Mujer }\end{array}$ & 77 & $21,75 \%$ & 109 & $16,59 \%$ & 100 & $15,22 \%$ \\
\hline $\begin{array}{l}\text { Autora Mujer } \\
\text { Director Hombre }\end{array}$ & 116 & $32,77 \%$ & 219 & $33,33 \%$ & 213 & $32,42 \%$ \\
\hline Total: & 354 & $100,00 \%$ & 657 & $100,00 \%$ & 657 & $100,00 \%$ \\
\hline
\end{tabular}

Fuente: Elaboración propia en base a Dialnet 2010-2020.

En lo que se refiere a las categorías temáticas y la orientación de la tesis a nivel dirección y codirección, tal y como se expone en la Tabla 12, se constata que las mujeres firman como autoras un mayor porcentaje de tesis en términos totales, 193 (54,52\%) frente a 161 $(45,48 \%)$. Una mayor presencia de mujeres que también se observa en todas las categorías temática salvo en Técnica y Tecnología, donde el $29,04 \%$ de autoras queda muy alejado del 70,96\% de tesis que son firmadas por sus compañeros hombres. En cualquier caso, solo en 
la categoría Comunicación, con un 60,22\%, las mujeres consiguen superar el 60\% del total de tesis firmadas en cada categoría. En el resto de los casos, este porcentaje oscila entre el 54,70\% en la categoría Arte y el 58,82\% en la categoría Sociología y Antropología.

Tabla 12 - Categorías temáticas en tesis sobre fotografía según el género de autores/as y del profesorado en la dirección/codirección

\begin{tabular}{|c|c|c|c|c|c|c|c|c|c|c|}
\hline $\begin{array}{l}\text { Categorías } \\
\text { temáticas }\end{array}$ & $\begin{array}{c}\mathrm{N}^{\circ} \\
\text { Tesis }\end{array}$ & $\begin{array}{l}\text { Autoría } \\
\text { Hombre }\end{array}$ & $\%$ & $\begin{array}{c}\text { Autoría } \\
\text { Mujer }\end{array}$ & $\%$ & $\begin{array}{c}\text { Total } \\
\text { Dir.Ce. }\end{array}$ & $\begin{array}{l}\text { Dir. Co. } \\
\text { Hombre }\end{array}$ & $\%$ & $\begin{array}{c}\text { Dir. Co. } \\
\text { Mujer }\end{array}$ & $\%$ \\
\hline Arte & 117 & 53 & $45,30 \%$ & 64 & $54,70 \%$ & 158 & 93 & $58,86 \%$ & 65 & $41,14 \%$ \\
\hline Comunicación & 88 & 35 & $39,78 \%$ & 53 & $60,22 \%$ & 118 & 73 & $61,86 \%$ & 45 & $38,14 \%$ \\
\hline $\begin{array}{l}\text { Autores } \\
\text { (vida y obra) }\end{array}$ & 57 & 25 & $43,85 \%$ & 32 & $56,15 \%$ & 75 & 38 & $50,67 \%$ & 37 & $49,33 \%$ \\
\hline Historia & 32 & 14 & $43,75 \%$ & 18 & $56,25 \%$ & 40 & 27 & $67,50 \%$ & 13 & $32,50 \%$ \\
\hline $\begin{array}{l}\text { Técnica } \\
\text { y Tecnología }\end{array}$ & 31 & 22 & 70,96 & 9 & $29,04 \%$ & 43 & 35 & $81,40 \%$ & 8 & $18,60 \%$ \\
\hline $\begin{array}{l}\text { Sociología } \\
\text { y Antropología }\end{array}$ & 17 & 7 & $41,18 \%$ & 10 & $58,82 \%$ & 21 & 16 & $76,20 \%$ & 5 & $23,80 \%$ \\
\hline $\begin{array}{l}\text { Documentación } \\
\text { y Archivo }\end{array}$ & 12 & 5 & $41,66 \%$ & 7 & $58,34 \%$ & 18 & 12 & $66,66 \%$ & 6 & $33,34 \%$ \\
\hline Totales & 354 & 161 & $45, \underline{48, \%}$ & 193 & $\mathbf{5 4 , 5 2} \%$ & 473 & 294 & $62,16 \%$ & 179 & $37,84 \%$ \\
\hline
\end{tabular}

Fuente: Elaboración propia en base a Dialnet. 2010-2020.

En lo que afecta a las propias temáticas dentro de cada categoría, no se aprecian diferencias significativas por género salvo en las tesis de Comunicación, donde las mujeres superan como autoras el número de tesis que abordan cuestiones de educomunicación, 14 tesis frente a las 9 firmadas por hombres. Asimismo, en la categoría Autores (vida y obra), donde las mujeres superan a los hombres en autoría, 32 tesis $(56,15 \%)$ frente a $25(43,85 \%)$, solo 13 de las 57 tesis contabilizadas abordan a una mujer fotógrafa, lo que supone un $22,80 \%$. Un conjunto de tesis en donde lo hombres superaran en número en dirección y codirección: 6 hombres frente a 5 mujeres.

Por otro lado, de esas 13 tesis que abordan de forma temática la vida y obra de mujeres fotógrafas, en 3 de ellas $(23,07 \%)$ el abordaje se realizará en diálogo con un fotógrafo. En solo 10 de 
36 La investigación académica sobre fotografía en España. Un análisis de las tesis doctorales ...

las 57 tesis de la categoría, lo que supone un 17,54\%, se tratará de forma completamente monográfica la figura de una mujer fotógrafa. Algo relevante cuando es, precisamente, la categoría con mayor porcentaje de mujeres en roles de dirección (49,33\%). Un porcentaje que convierte a la categoría Autores (vida y obra) en la única que roza la equidad en hombres y mujeres en dirección y codirección de tesis.

En cuanto a las 10 tesis doctorales que abordan el estudio monográfico de una mujer fotógrafa, 8 de ellas (el 80\%) serán firmadas por mujeres y solo 2 por hombres (el 20\%). Relevante será también la nacionalidad de las fotógrafas en el total de la categoría. En solo 1 de las 57 tesis (el 1,75\%), se abordará a una fotógrafa española, en este caso fotoperiodista. Una tesis cuya autoría corresponde a un hombre y estará, al tiempo, dirigida por una mujer como directora.

Por último, será relevante la constatación de la amplia presencia de hombres en la dirección y codirección de tesis en la categoría Técnica y Tecnología que, con un $81,40 \%$, supone el porcentaje más alto $\mathrm{y}$, al tiempo, muestra un desequilibrio similar en el porcentaje de autoría de las tesis en su misma categoría: un $70,96 \%$ en autores hombres frente al $29,04 \%$ de tesis firmadas por mujeres. Un hecho que, siendo el más destacado en porcentaje, será la única excepción en cuanto a autoría. En el resto de categorías, pese a seguir presentando un porcentaje más alto en número de hombres en la dirección y codirección de tesis, en la autoría de las tesis doctorales serán siempre las mujeres las que superen a los hombres en número. 


\section{Conclusión}

La investigación académica sobre fotografía que la universidad española ha llevado a cabo a través de tesis doctorales entre 2010 y 2020 ha experimentado una tendencia marcadamente ascendente respecto a los datos d e producción de tesis ofrecidos por estudios previos. De las 239 investigaciones doctorales leídas entre 1976 y 2010 según los datos ofrecidos por Sánchez-Vigil, MarcosRecio y Olivera-Zaldua (2014), solo en la siguiente década el número de tesis ha ascendido a 354 tesis, lo que supone un aumento significativo no solo en número, sino también en el interés y grado de aceptación de este objeto de estudio. Esta tendencia al alza, al hilo de los datos ofrecidos por Olivera-Zaldua, Sanchez-Vigil y Marcos-Recio (2016), experimenta su pico más alto entre 2015 y 2017, coincidiendo con la reforma de los planes de tercer ciclo de la universidad española y la implantación del Plan Bolonia, que aceleró la defensa de tesis doctorales en curso que estaban adscritas a planes de doctorado a extinguir. Asimismo, este incremento en el número de tesis defendidas entre 2010 y 2020 es coincidente, e incluso menos acusado, con el que se produce en el área de Ciencias Sociales y Humanidades. Por otro lado, y como ocurre de forma especial en el área de Ciencias Sociales, 2020 será un año donde menos tesis doctorales sobre fotografía se registran. Un descenso que puede deberse tanto al aplazamiento de las defensas con motivo de la pandemia de la covid-19 como al retardo en los registros de las tesis en las bases de datos.

La multiplicidad de enfoques y la diversidad en el objeto 
de estudio del corpus de tesis doctorales sobre fotografía no solo señalan la dificultad, y su consiguiente sesgo, a la hora de establecer categorías temáticas lo suficientemente integradoras. También indican la riqueza de este ámbito de estudio y la transversalidad que ofrece el uso y los usos de la fotografía en una amplia variedad facultades que acogen este tipo de temáticas en sus planes de doctorado. En ese sentido, que 473 docentes hayan participado en tareas de dirección y codirección de las 354 tesis objeto de estudio y que, al tiempo, el $87 \%$ de este profesorado tan solo ha dirigido o codirigido una tesis doctoral sobre fotografía, confirma la versatilidad de la temática y la posibilidad de llevar a cabo distintos abordajes desde distintos ámbitos.

La universidad española con mayor número de tesis sobre fotografía es la Complutense de Madrid (61), que también lidera en número de tesis en el área de Humanidades, seguida de la Universitat de Barcelona (30), la Universidad de Granada (26), la Politècnica de València (22) y la Universidad de Sevilla (22). Las dos primeras siguen liderando el número de tesis sobre fotografía desde 1976 siendo destacable la irrupción de la Universidad de Granada en tercer lugar y el retroceso de la Politècnica de Valencia del tercer al cuarto puesto en esta última década atendiendo a los datos de 1976 a 2010 ofrecidos por Sánchez-Vigil, Marcos-Recio y Olivera-Zaldua (2014).

En cuanto a los centros que acogen un mayor número de tesis, destacarán las facultades del área de Humanidades y las Ciencias Sociales lo que confirma el predominio de estas áreas de conocimiento a la hora de abordar la investigación sobre fotografía. No obstante, la presencia de tesis en la E.T.S. Arquitectura (22), en el ámbito 
de la Ingeniería, Informática y TIC (8), Física (2) o Medicina (2) plantea un interesante horizonte que merece ser observado a futuro. Un escenario que da como resultado el siguiente número de tesis en función de las categorías temáticas: Arte (117); Comunicación (88); Autores (vida y obra) (57); Historia (32); Técnica y Tecnología (31); Sociología y Antropología (17); Documentación y Archivo (12).

El análisis desde una perspectiva de género del conjunto tesis en cuanto autoría confirma una mayor presencia de mujeres autoras $(54,52 \%)$ que de autores (48,48\%). Porcentaje que varía significativamente en cuanto al profesorado en la dirección y codirección donde se constatan 294 docentes hombres $(62,16 \%)$ frente a 179 mujeres $(37,84 \%)$. Una desigualdad que se observa también en la relación entre autoría y profesorado en la dirección de tesis. Las relaciones entre autor y autora con un director hombre se mantienen en unos parámetros más estables $(36,44 \%$ y $32,77 \%$ respectivamente) que en los casos donde la dirección o codirección corresponde a una mujer, donde la diferencia es mucho más significativa. En este sentido, se confirma que la relación "autor hombre y directora mujer" es mucho menos frecuente, 32 tesis $(9,04 \%)$, que la que la relación "autora mujer y directora mujer", que asciende a 77 tesis $(21,75 \%)$ en el periodo objeto de estudio.

Por otro lado, la gran dispersión de docentes en roles de dirección y codirección de tesis en fotografía con muy pocas tesis acumuladas en su haber, habla de una escasa especialización y de un modelo académico débil para garantizar a largo plazo una producción académica sólida sobre fotografía a nivel investigación doctoral. Más todavía en cuanto a investigación dobre mujeres fotógrafas. Las autoras destacan en las tesis sobre Autores (vida 
40 La investigación académica sobre fotografía en España. Un análisis de las tesis doctorales ...

y obra) desempeñando un papel clave a la hora de mantener una tradición investigadora centrada de forma monográfica en mujeres fotógrafas. En ese sentido, las mujeres investigadoras parecen haber asumido la responsabilidad de que la memoria de estas fotógrafas no quede en el olvido.

Afrontar, por tanto, el estudio de la producción científica de tesis doctorales sobre fotografía llevadas a cabo en la universidad española incorporando la perspectiva de género se convierte en una necesidad urgente y necesaria no solo para atenerse a las recomendaciones de la sección $\mathrm{J}$ de la Declaración y la Plataforma de Acción de Beijing 95 (DECLARACIÓN..., 1995). Es, a la vista de los resultados obtenidos entre 2010 y 2020, fundamental para seguir comprendiendo cómo se articula la investigación académica en el sistema universitario español y dar visibilidad a la investigación realizada por mujeres en aras de conseguir una igualdad real y efectiva en el acceso y ascenso en la carrera científica e investigadora.

\section{Referencias}

DECLARACIÓN y plataforma de acción de Beijing. En: CONFERENCIA MUNDIAL SOBRE LA MUJER, 4., 1995, Beijing. [Beijing]: ONU Mujeres, 1995. pdf.

FONTCUBERTA, Joan. Apéndice. Notas sobre la fotografía española. En: NEWHALL, Beaumont. Historia de la fotografía: desde sus orígenes hasta nuestros días. Barcelona: Gustavo Gili, 1983. p. 300-322.

GARCÍA-RAMOS, Francisco-José. Relatos y representaciones de la mujer fotoperiodista en la prensa y el cine español en los años 50 
y 60: el caso de la fotógrafa Juana Biarnés. Revista Prisma Social, Madrid, España, n. esp. 2, p. 126-166, 2017.

GARCÍA-RAMOS, Francisco-José; JIMÉNEZ-GÓMEZ, Isidro. La (in)visibilidad de las fotógrafas publicitarias en España: un estudio a través de su presencia en Ads of the world (2005-2018). En: GARCÍA-RAMOS, Francisco-José; FELTEN, Uta. Fotografía (femenino; plural): visiones, ensayos y otros escritos sobre mujeres fotógrafas. Madrid: Fragua, 2019. p. 229-252.

GARCÍA-RAMOS, Francisco-José; ZURIAN, Francisco; NÚÑEZ-GÓMEZ, Patricia. Gender studies in communication degrees. [Los estudios de género en los Grados de Comunicación]. Comunicar: Revista Científica de Comunicación y Educación, Huelva, España, v. 28, n. 63, p. 21-30, 2. trim., abr. 2020. DOI: https://doi. org/10.3916/C63-2020-02.

JONES, Daniel E.; BARÓ I QUERALT, Jaume. Investigación sobre comunicación en España: aproximación bibliométrica a las tesis doctorales (1926-1998). Barcelona: ComCat, 2000.

LEÓN MARIN, Joaquín; MAGRIÑÁ CONTRERAS, Marta. Dialnet, una hemeroteca virtual de revistas hispanas sobre la base de la cooperación bibliotecaria. EI Profesional de la Información, Barcelona, v. 13, n. 4, p. 281-283, 2004.

MÉNDEZ-RODRÍGUEZ, Luis; PLAZA-ORELLANA, Rocío. Teaching photography in the 21st century: old problems and new needs. Field study in the area of Art History: sources, methodologies and digital culture. En: CONGRESO INTERNACIONAL SOBRE FOTOGRAFÍA, 2017, Valencia, España. Actas [...]. Valencia: Universitat Politècnica de València, 2017. p. 573-585.

MOREIRO-GONZÁLEZ, Carlos Javier. La cooperación internacional y europea para rescatar la memoria audiovisual. Revista Ge- 
42 La investigación académica sobre fotografía en España. Un análisis de las tesis doctorales ...

neral de Derecho Europeo, Madrid, v. 22, p. 1-33, 2010.

OLIVERA ZALDUA, María. La fotografía más allá de una disciplina: pasado, presente y futuro. En: MARCOS-RECIO, Juan Carlos; SÁNCHEZ-VIGIL, Juan Miguel. Del artefacto mágico al pixel: estudios de fotografía. Madrid: Universidad Complutense, 2014. p. 193-201.

OLIVERA-ZALDUA, María; SÁNCHEZ-VIGIL, Juan Miguel; MARCOS-RECIO, Juan Carlos. Análisis de las tesis doctorales sobre fotografía en la universidad española (enero de 2013-marzo de 2016). Ibersid: revista de sistemas de información y documentación, Zaragoza, Spain, v. 10, n. 2, p. 13-20, 2016.

RIEGO, Bernardo. La fotografía como documento. En: RIEGO, Bernardo; SÁNCHEZ-GÓMEZ, Miguel Ángel; SOUGEZ, Marie-Loup. La fotografía y sus posibilidades documentales. Una introducción a su utilización en las Ciencias Sociales. Santander: ICE-Universidad de Cantabria, 1989.

RIEGO, Bernardo. La fotografía en la enseñanza universitaria: hacia un futuro sin apenas pasado. En: DOCTOR, Rafael; BONET, Juan Manuel; SOUGEZ, Marie-Loup; LOBATO, Rafael Sanz;

LEVENFELD, Rafael; PÉREZ MÍNGUEZ, Pablo, RUEDA, Jorge. Nueva Lente: inicio y desarrollo de la fotografía de la creación en España. Jornadas de Estudio. Madrid: Dirección General de Patrimonio Cultural, Consejería de Educación y Cultura, 1993. p. 203214.

SÁNCHEZ-VIGIL, Juan Miguel; MARCOS-RECIO, Juan Carlos; OLIVERA- ZALDUA, María. Tesis doctorales sobre fotografía en la universidad española: análisis de la producción y dirección (1976-2012). Revista Española de Documentación Científica, Madrid, España, v. 37, n. 1, e034, 2014. DOI: http://dx.doi. 
org/10.3989/redc.2014.1.1073.

VÁZQUEZ-CASILLA, José Fernando. La historia y la fotografía en España (1975-1999). Estado de la cuestión. En: CONGRESO DE HISTORIA DE LA FOTOGRAFÍA, 3., 2007, Zarautz. Actas [...]. Zarautz: Photomuseum, 2008. p. 215-227.

VEGA DE LA ROSA, Carmelo. Buscando modelos: la historia de la fotografía en España, 1981-2006. Revista Latente, San Cristóbal de La Laguna, España, v. 5, p. 27-56, 2007.

VEGA DE LA ROSA, Carmelo. La fotografía en la Universidad, ¿una historia con futuro? Revista Latente, San Cristóbal de La Laguna, España, v. 6, p. 47-52, 2008.

VEGA PÉREZ, Celia. Los estudios de fotografía en la universidad. En: MARCOS-RECIO, Juan Carlos; SÁNCHEZ-VIGIL, Juan Miguel. Del artefacto mágico al pixel. Estudios de fotografía. Madrid: Universidad Complutense, 2014. p. 227-239. 